\title{
PRIVATIZACIÓN DEL SISTEMA DE SEGURIDAD SOCIAL EN MÉXICO: UN ESTUDIO DE LA VULNERACIÓN A LOS DERECHOS HUMANOS
}

\section{PRIVATIZATION OF SOCIAL SECURITY IN MEXICO: A STUDY OF HUMAN RIGHTS' INFRINGEMENT}

\author{
Dr. Valentín Armenta Ramírez ${ }^{1}$ \\ Benemérita Universidad Autónoma de Puebla \\ ORCID: 0000-0002-4604-9307 \\ valentin.armenta@correo.buap.mx \\ María de Lourdes Castellanos Villalobos ${ }^{2}$ \\ Universidad Veracruzana \\ ORCID: 0000-0002-6358-3413 \\ locastellanos@uv.mx \\ Ernesto Levet Gorozpe ${ }^{3}$ \\ Universidad Veracruzana \\ ORCID: 0000-0002-8182-572X \\ elevet@uv.mx \\ México
}

\section{SUMARIO}

- Introducción

- Sistema de ahorro para el retiro (privatización)

- Riesgo de la privatización de pensiones en México

- Conclusión

- Fuentes de información

\section{RESUMEN}

El artículo basa su estudio en el método científico. El estudio utiliza la observación, experimentación y comprobación para la eficacia del método científico. La hipótesis a desarrollar es ¿con la privatización de las pensiones, se vulneran derechos humanos? En este sentido, se arriba a que la privatización de pensiones en México es un

\footnotetext{
${ }^{1}$ Doctor en Derecho, Maestro en Derecho con Terminal en Fiscal, Maestro en Derecho y Ciencias Penales; y, Licenciado en Derecho. Profesor investigador en la Benemérita Universidad Autónoma de Puebla, adscrito a la Facultad de Derecho y Ciencias Sociales. Correo institucional: valentin.armenta@ correo.buap.mx

${ }^{2}$ Doctor en Gobierno y Administración Pública, Maestría en Administración y Políticas Públicas con especialidad en Ciencias Políticas y Licenciado en Derecho por la Universidad Veracruzana. Adscrita al Sistema de Enseñanza Abierta (SEA) de la Universidad Veracruzana. Representante de la Unidad de Género en el SEA y Coordinadora de la Academia de Derecho Procesal. Correo institucional: locastellanos@uv.mx

${ }^{3}$ Profesor Tiempo Completo de la Universidad Veracruzana con maestría en Administración y Políticas Públicas con Especialidad en Ciencias Políticas. Miembro del Núcleo Académico Básico (NAB) de la Maestría en Derechos Humanos y Justicia Constitucional avalada por CONACYT. Consejero Maestro por el programa educativo de Derecho. Correo institucional: elevet@uv.mx
} 
riesgo para los trabajadores de este país, existe vulneración de sus derechos humanos para lograr una pensión al final de sus días laborales. El Estado Mexicano a finales de los noventas, en específico 1997, adoptó el sistema de capitalización individual para el tema de pensiones, esto es, que el recurso destinado a las pensiones lo administra la iniciativa privada mediante Administradoras de Fondos para el Retiro. Sin embargo, al tratar de aumentar el ahorro para el retiro en beneficio de los trabajadores, los recursos se invierten a través de Sociedades de Inversión Especializadas en Fondos de Retiro. En consecuencia, es peligroso que la iniciativa privada administre en su totalidad ese fondo, pues en 2018 se publicó "Cuatro afores invierten en el nuevo aeropuerto" (Albarrán, 2018) y meses después se canceló el proyecto, pero al parecer de nueva cuenta dicho proyecto podría iniciarse. Por consiguiente, dejar todos los recursos de las pensiones en manos de instituciones financieras es un riesgo para los trabajadores. Se debe recordar que a partir de la crisis financiera mundial de 2008, se generó pérdidas (minusvalías para la Ley de los Sistemas de Ahorro para el Retiro) en el Sistema de Ahorro para el Retiro.

\section{ABSTRACT}

The article in question, based its study on the scientific method. The study uses observation, experimentation and verification for the effectiveness of the scientific method. The hypothesis to be developed is if with the privatization of pensions, human rights are violated? In this sense, it is arrived at that the privatization of pensions payments in Mexico is a risk for the workers of this country, it violates their human rights to obtain a pension at the end of their working days. The Mexican State at the end of the nineties, specifically 1997 , adopted the system of individual capitalization for the issue of pensions, that is, that the resource for pensions payments is administered by the private initiative through fund managers for the Retirement. However, in trying to increase retirement savings for the benefit of workers, resources are invested through Specialized Investment Companies in Retirement Funds Consequently, it's risk to the private companies to administer that fund, because, in 2018, "Four Afores invest in the new airport" published and months later the project canceled, but apparently that project could begin again. Therefore, leaving all pension resources in the hands of financial institutions is a risk for workers, we must remember that from the global financial crisis in twenty o eight, it generated losses (losses for the Savings Systems Law for Retirement) in the Retirement Savings System.

\section{PALABRAS CLAVE}

Administradoras de Fondos para el Retiro (Afores), Pensión, Sistema de Ahorro para el Retiro, Sociedades de Inversión especializadas en Fondos para el Retiro (Siefores), Trabajador, Derecho Humano

\section{KEYWORDS}

Administrators of Retirement Funds (Afores), Pension payment, Retirement Savings System, Investment Companies specialized in Retirement Funds (Siefores), Worker, Human Rights.

\section{INTRODUCCIÓN}

El presente artículo evidenciará el riesgo de tener sólo pensiones privadas en México, lo cual generará vulneración a los Derechos Humanos (DDHH), independiente a que en la Convención Americana sobre Derechos Humanos (CADH) suscrita en la Conferencia Especializada Interamericana sobre Derechos Humanos del Pacto de San José numeral 26, establece el derecho a la seguridad social. 
Entonces, el Estado está obligado por el pacto antes citado a generar esa seguridad social (en específico pensiones), sin embargo, ante la falta de una garantía que logre ese cumplimiento, puede deslindarse y omitir el pago de pensiones en una cuestión formal al decir nada tiene que ver desde mil novecientos noventa y siete en el cambio de sistema.

Asimismo, el Sistema de Ahorro para el Retiro (SAR), el cual se basa en una capitalización individual, carece de garantía para los gobernados, pues pueden darse dos supuestos, el primero es la cantidad de pérdidas en el dinero invertido que se refleja en el fondo de ahorro para el retiro; y, el segundo, por la inversión de dichos fondos de ahorro de los trabajadores en proyectos del Estado como el Nuevo Aeropuerto Internacional de la Ciudad de México (NAICM).

Entonces, durante el desarrollo del presente artículo, se demostrarán algunas pérdidas al Sistema de Ahorro para el Retiro (SAR) a partir de 2008, además del riesgo de invertir los recursos en el mercado de valores y sólo contar con un sistema privado de pensiones, lo que generará que la pensión deje de ser un derecho humano. Por lo cual, una figura importante o adecuación que requiere el Estado Mexicano es instaurar una pensión pública como sucede en Perú, con la finalidad de garantizar la pensión del trabajador, pues al tener el Estado parte del fondo de ahorro para el retiro, puede garantizar pensiones.

\section{SISTEMA DE AHORRO PARA EL RETIRO (PRIVATIZACIÓN)}

Para iniciar con el tema del Sistema de Ahorro para el Retiro (SAR), fue necesario que la Carta Magna hablara de una seguridad social, después de una etapa revolucionaria:

La Revolución Francesa y la Declaración de Independencia de los Estados Unidos a fines del siglo XVIII provocaron que se creara una conciencia clara de las posibilidades de organización colectiva de los diversos grupos sociales que atestiguaron el surgimiento de nuevas estructuras y formas de gobierno (Meléndez George, 2011, pág. 39 y 40).

Luego, en México con sucesos como las huelgas de Río Blanco y Cananea, ocasionó que el 5 de febrero de 1917 entrara en vigor la Constitución Política de los Estados Unidos Mexicanos, de este modo, el artículo 123 desde ese momento habló como hasta la fecha de la previsión social, pero la fracción importante que inició el tema de pensiones, se abordó en el apartado A, fracción XXIX (Congreso Federal, 1917) y en dicha fracción se consideró inculcar la previsión social. Luego, existió una reforma a la fracción antes citada en 1929 (Congreso Federal, 1929), la cual ya contemplaba la expedición de la Ley del Seguro Social (LSS); y, una nueva reforma en 1943, en la que se consideró de utilidad pública a la Ley del Seguro Social, la misma que contempló seguros de invalidez, vejez, vida, cesación involuntaria del trabajo, de enfermedades y accidentes, como hasta la fecha está plasmada.

Entonces, "la Constitución Mexicana de 1917, es el resultado de un movimiento social basado en ideas avanzadas con principios democráticos" (Gutiérrez Ayala, 2017, pág. 89), esos movimientos y las ideas avanzadas fueron la antesala para el artículo 123 de la Carta Magna.

Con lo anterior, el origen de la seguridad Social en México se originó en 1917, a partir de ese momento como varios países en la actualidad del continente europeo, México tenía un sistema solidario para generar las pensiones, el Estado administraba ese ahorro, pero en 1997, tras varias reformas al Sistema de Ahorro para el Retiro (SAR), el sistema se convirtió en un mecanismo de capitalización individual. 
Es así que surgieron figuras como las Administradoras de Fondos para el Retiro (en adelante, AFORES) y Sociedades de Inversión Especializadas en Fondos para el Retiro (en adelante, SIEFORES), a partir de ese momento los recursos que tenía el Estado a través del Instituto Mexicano del Seguro Social (en adelante, IMSS), pasaron a las AFORES con la finalidad de administrar y generar una pensión para cada trabajador en México, con la ideología que al administrar un recurso, se aumentara ese ahorro.

En consecuencia, la Ley de los Sistemas de Ahorro para el Retiro (LSAR), desde su creación se encargó de regular y vigilar el Sistema de Ahorro para el Retiro (SAR), se apoyó con la Ley del Seguro Social (Congreso Federal, 1995), la cual, en el artículo 11, establece el régimen obligatorio y contempla seguros para: riesgos de trabajo; enfermedades y maternidad; Invalidez y vida, retiro, cesantía en edad avanzada y vejez; $y$, guarderías $y$ prestaciones sociales.

\section{RIESGO DE LA PRIVATIZACIÓN DE PENSIONES EN MÉXICO}

En el punto anterior se comentó el surgimiento de la seguridad social, en específico, lo relativo al derecho a la pensión y, por ende, desde 1997 el Sistema de Ahorro para el Retiro (en adelante SAR), sistema que se basa en la capitalización individual. En consecuencia, al momento del cambio del sistema, IMSS que tenía los recursos de los trabajadores, quienes optaron por el cambio de sistema por una versión en la cual su ahorro crecería y al momento de su retiro su pensión sería mejor a que la dejaran en el sistema anterior, los transfirió a las AFORES, con ello los recursos pasaron a la iniciativa privada.

Al pasar los recursos a la iniciativa privada se genera la capitalización individual, fue un modelo aceptado en América, tal y como lo comentó Sergio Kurczyn, quien señala que "entre los principales argumentos para la reforma se encuentran la inviabilidad financiera del anterior sistema" (Kurczyn Bañuelos, 1996, pág. 741), ejemplo de lo anterior es el modelo del Estado Chileno, pionero en el sistema.

También Perú adoptó el sistema de capitalización individual, sin embargo, consideró dejar un sistema público de pensiones, el cual carece de un crecimiento alto del ahorro para la pensión como lo vende la iniciativa privada, pero es un sistema seguro, debido a que el Estado garantizará a sus gobernados la pensión y jamás dejarlos a la deriva como puede pasar con el sistema de capitalización individual, por medio de las AFORES.

Entonces, México inició el sistema de capitalización individual y fue en 2008 que existió una crisis mundial financiera: "Funcionarios, políticos y banqueros son los culpables del colapso económico experimentado en 2008 en Estados Unidos, asegura un informe de la Comisión Investigadora de la Crisis Financiera de EE.UU..." (BBC Mundo, 2011, párrafo primero), dicha crisis evidenció en México pérdidas en la Ley de los Sistema de Ahorro para el Retiro (minusvalías), pues la AFORE envío recursos para inversión a las SIEFORES y éstas invierten el recurso con la finalidad de incrementar el ahorro de manera aparente.

En consecuencia, una vez que el recurso destinado por las AFORES a las SIEFORES se invierte, queda en espera de que las acciones suban y crezca el ahorro; sin embargo, al existir la crisis antes citada, se evidenciaron pérdidas en el SAR lo cual generó la duda del sistema, muestra de ello son las siguientes publicaciones en torno al tema, que dan evidencia de lo ya asentado, por consiguiente: algunas pérdidas que se suscitaron entre 2007 y 2009 fueron: 
Pérdidas en los ahorros de trabajadores por 147,850 millones de pesos en 23 meses

La Comisión Nacional del Sistema de Ahorro para el Retiro reconoció ante la Cámara de Diputados que de marzo de 2007 a febrero de 2009 las pérdidas en los ahorros de los trabajadores mexicanos, inscritos en las administradoras de fondos para el retiro (Afore) sumaron $147 \mathrm{mil}$ 850 millones de pesos.

De diciembre de 2008 a febrero del presente la merma fue de 43 mil 546 millones de pesos.

En contraste, en 2007 las Afore obtuvieron ganancias por 15 mil 93 millones de pesos... (Garduño, 2009, Título, párrafo primero y tercero)

Luego, el 7 de julio de 2013:

Afores, con las minusvalías más grandes de la historia

Las administradoras de fondos de ahorro para el retiro (afores) registraron su segundo mes consecutivo de minusvalías durante este $2013 \ldots$ Al cierre de junio, los recursos que las afores administran cayeron en 74,424 millones de pesos, para llegar a un total de activos de 1 billón 919,494 millones de pesos, dichos recursos son $3.9 \%$ más bajos que los reportados en mayo, cuando el saldo era de 1 billón 994,918 millones de pesos. (Torres, El Economista, 2013, Título, párrafo segundo)

Posterior a 2013, en marzo de 2015:

PÉRDIDAS

MULTIMILLONARIAS AFORES
* Las Afores Han Perdido Alrededor de 400 Mil Millones de Pesos de los Trabajadores... volvieron a tener pérdidas millonarias las cuentas de ahorro para el retiro de los trabajadores (Afores), administradas por la banca privada en México. Durante el mes de marzo del 2015 las pérdidas fueron por 33 mil 608 millones de pesos, de acuerdo al informe de la Comisión Nacional del Sistema de Ahorro para el Retiro (CONSAR)... (Martinez Gonzalez, 2015, párrafo primero)

El de noviembre de 2018:

Sufren afores las peores minusvalías del Sexenio

Registraron minusvalías por $131 \mathrm{mil}$ 649 millones de pesos en octubre... (Carrillo, 2018, párrafo primero).

El veinte de mayo de dos mil diecinueve:

Las afores con mayores pérdidas de cuentas de ahorro al cierre de abril, según la CONSAR.

De las 10 Administradoras de Fondos para el Retiro que participan en el Sistema de Ahorro para el Retiro, siete mostraron salida de cuentas $y$ tres fueron las beneficiadas por esos cambios... (NOTIMEX, 2019, párrafo primero).

En relación a la primera caída entre 2007 y 2009, lo externaron los medios de información y lo mejor es que se pudo corroborar en Estados de Cuenta de los derechohabientes, el ejemplo es "Gregorio X", quién en su cuenta de ahorro para el retiro tuvo pérdidas, pues entre enero y febrero de 2008 tenía $\$ 231,352.97$ y en el siguiente estado de cuenta del mes de julio y agosto del mismo año tuvo \$208,516.87: 


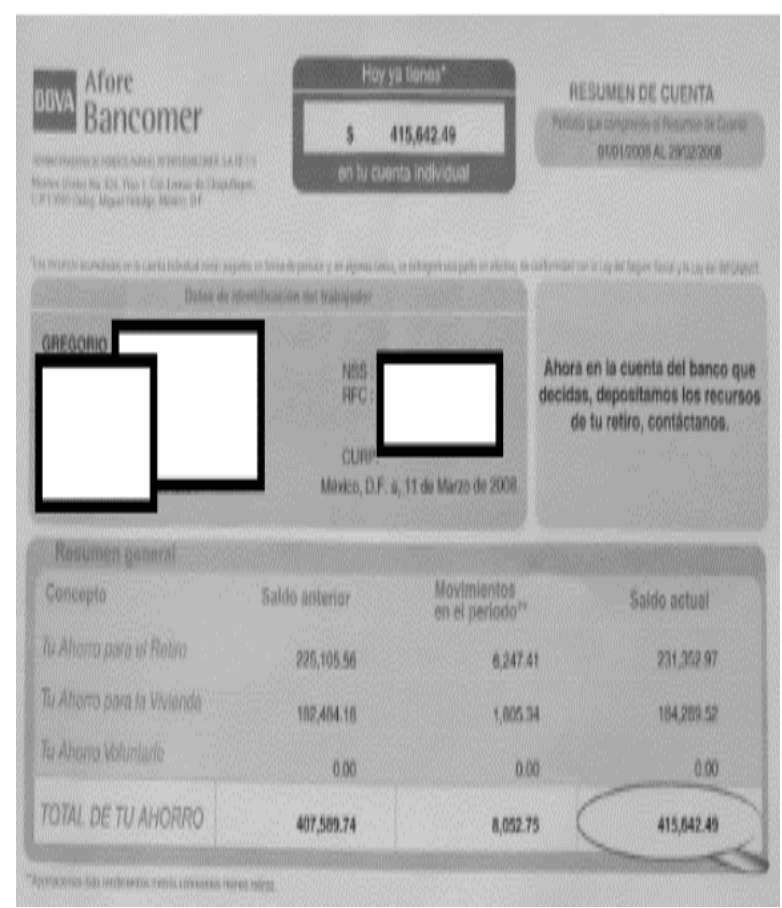

Imagen 1

Estado de Cuenta Marzo 2008

Fuente: Archivo personal

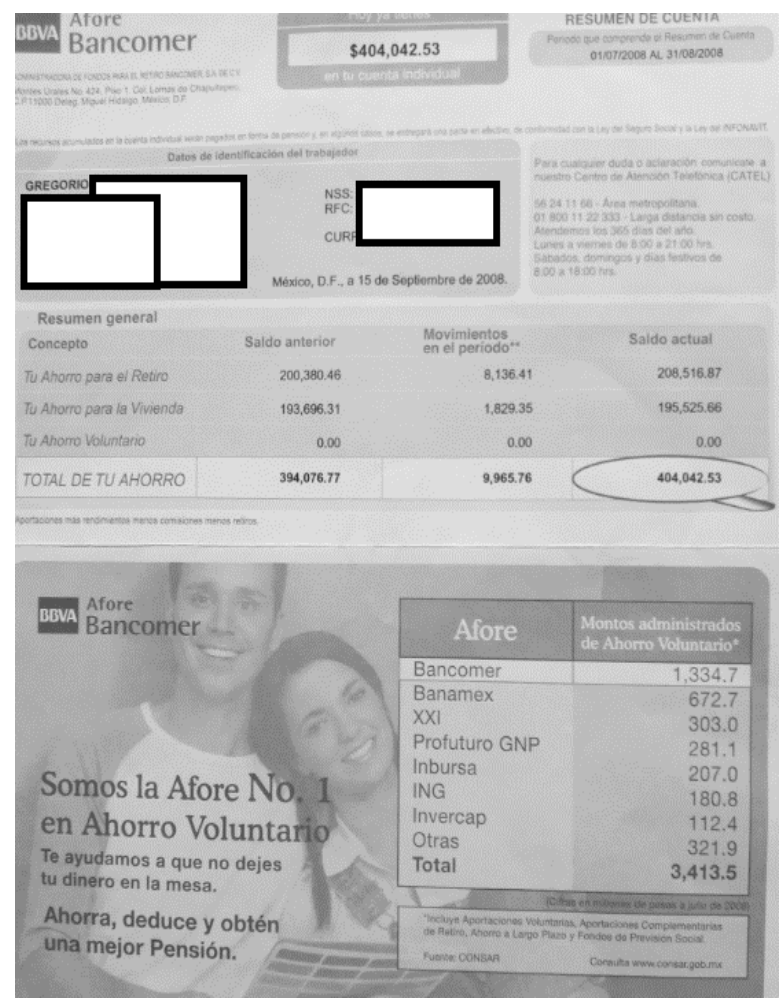

Imagen 2

Estado de Cuenta Septiembre 2008

Fuente: Archivo personal
En consecuencia, resulta aterrador realizar un cálculo de cuánto se perdió en un total de las AFORES en ese año, pues si hacemos un cálculo aproximado, si "Gregorio X" tenía $\$ 231,352.97$ y en el siguiente estado de cuenta del mismo año \$208,516.87, entonces, la pérdida fue de $\$ 22,836.1$, pérdidas derivadas del ahorro de los trabajadores, en 2008.

Posterior a 2008, como se estableció antes en otra noticia de este artículo, en 2013 también hubo una caída muy fuerte en el Sistema de Ahorro para el Retiro (SAR), por lo cual, "Gregorio X" proporcionó los documentos, el primero del periodo de enero al mes de abril, y el segundo de mayo al mes de agosto, ambos de 2013, en los cuales constan dichas pérdidas:

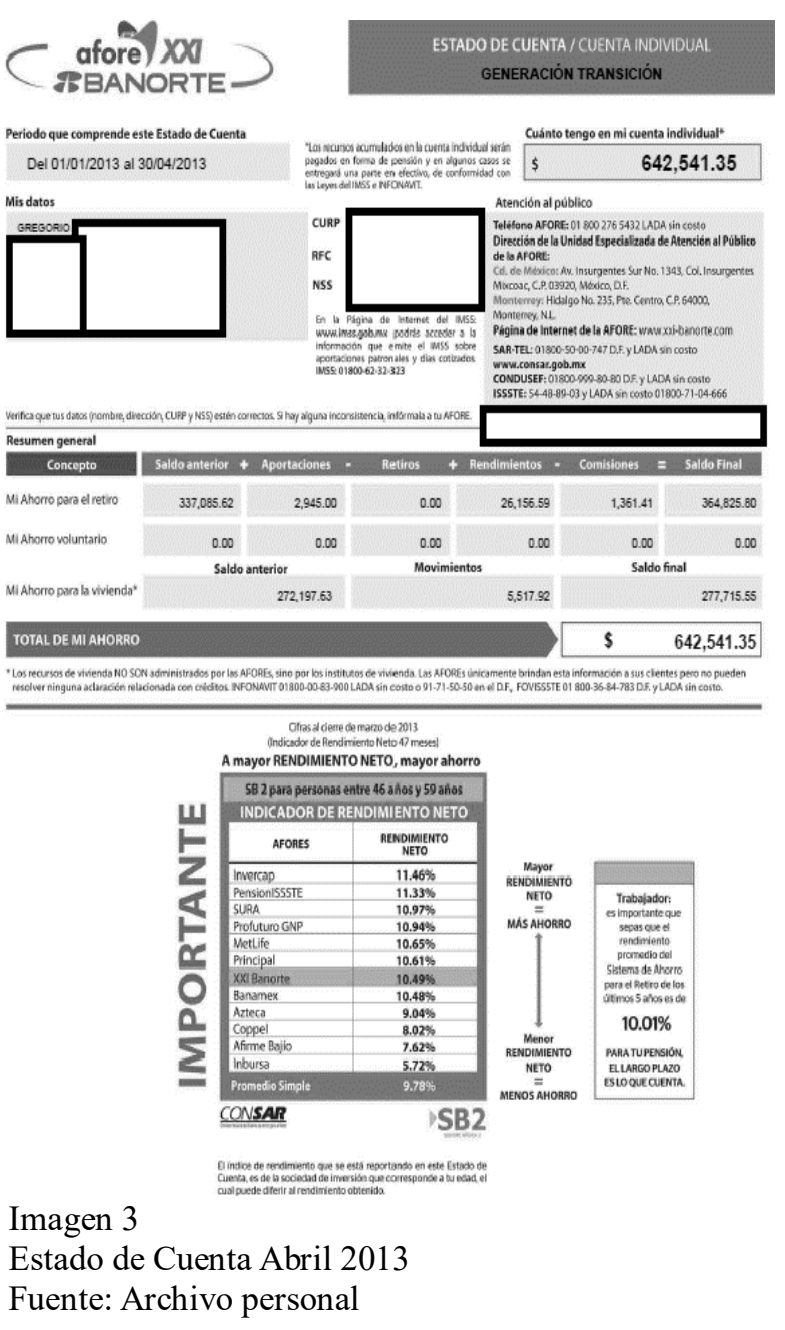




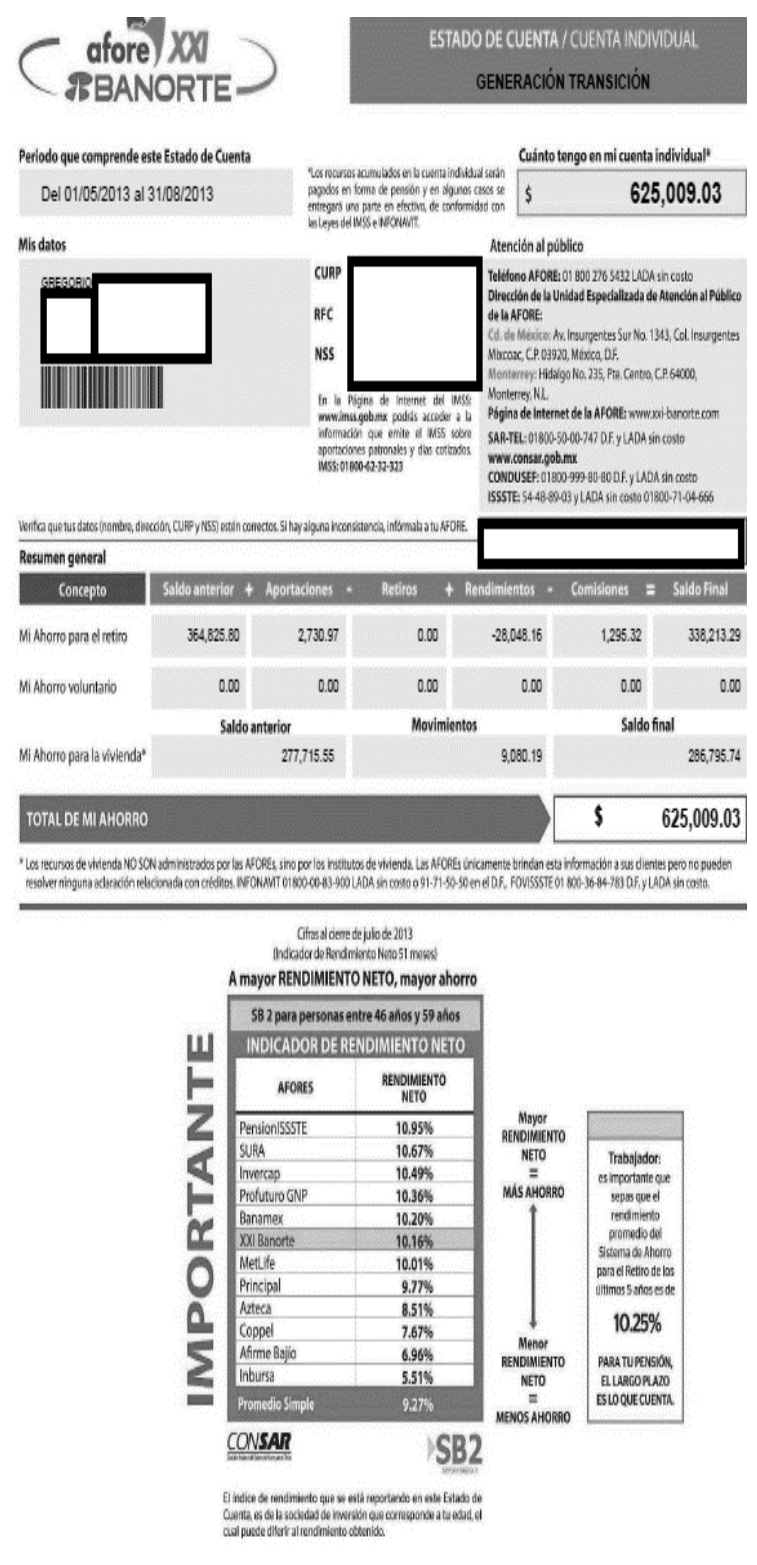

Imagen 4

Estado de Cuenta Agosto 2013

Fuente: Archivo personal

Por consiguiente, las pérdidas para “Gregorio X” en 2013 fueron de \$26,612.51, si se realizara una multiplicación de los derechohabientes con las pérdidas, sería aterrador, pues, en 2012, la Afore XXI Banorte en la cual desde 2013 estuvo "Gregorio X", ocupó el lugar de la administradora más grande del Sistema de Ahorro para el Retiro en enero de 2012, al pasar de 3.1 millones de clientes a 11.2 millones. (Sandoval, 2018). Por tanto, en ese año existieron grandes pérdidas al
Sistema de Ahorro para el Retiro como se estableció antes en las notas de los medios de información y se evidencia en los documentos de los Estados de Cuenta.

A fin de dar certeza a la investigación, a"Gregorio X" se le realizó una entrevista cerrada en la cual respondió tres preguntas de la siguiente manera:

1. ¿Conoce el Sistema de Ahorro para el Retiro vigente a partir de mil novecientos noventa y siete?

Respuesta. Lo conozco ahora que he investigado, pero cuando cambió el sistema de 1973, nos prometieron que si nos cambiábamos al actual sistema ahorraríamos más, pero por lo que analizo es falso.

2. Con lo que tiene ahorrado en la actualidad ¿sabe de cuánto será su pensión? Respuesta. He preguntado en el banco y por mi salario, será aproximado de dos mil quinientos pesos.

3. ¿Le da certeza el actual Sistema de Ahorro para el Retiro?

Respuesta. La verdad me arrepiento de estar en este sistema de capitalización individual, pues en algunos meses sube mi ahorro para el retiro y en otra baja

Para continuar con el presente artículo, resulta necesario evidenciar que hasta 2018 existen alrededor de sesenta millones de derechohabientes en México y su cuenta la maneja alguna de las AFORES, destaca que pensión ISSSTE se omite en el cálculo, entonces si suponemos que en promedio que cada derechohabiente pierde $\$ 10.00$ (diez pesos) en su cuenta de retiro, el total que se pierde en el Sistema de Ahorro para el Retiro es de $\$ 600,000,000.00$ (seiscientos millones de pesos). Con lo anterior, es obvio que el Estado vulnera la pensión del trabajador al dejar todo en manos de la iniciativa privada, pues cuando ganan dividendos nunca demuestran todo lo que 
ganan estas empresas, por lo cual, es un negocio redondo, porque manejan $\mathrm{e}$ invierten un dinero que es del trabajador y en las pérdidas pierden los trabajadores $\mathrm{y}$ cuando ganan sólo les dan una comisión a quienes arriesgan su dinero todos los días. En la siguiente imagen se plasma el número de derechohabientes con la finalidad de dar certeza al cálculo realizado en el presente párrafo:

\begin{tabular}{|c|c|c|c|}
\hline \multirow{2}{*}{ AFORE } & \multicolumn{2}{|c|}{ CUENTAS } & \multirow{2}{*}{ VALIDACIÓN } \\
\hline & Enero & Octubre & \\
\hline Azteca & $2,207,740$ & $11,604,548$ & $9,396,808$ \\
\hline Citibanamex & $10,716,326$ & $11,716,549$ & $1,000,223$ \\
\hline Coppel & $8,872,333$ & $10,271,467$ & 1,39,134 \\
\hline Inbursa & $1,059,699$ & $1,046,422$ & $-13,277$ \\
\hline Invercap & $2,385,236$ & $2,020,320$ & $-364,916$ \\
\hline PensionISSSTE & $2,082,646$ & $2,083,350$ & 704 \\
\hline Principal & $2,690,439$ & $3,028,429$ & 337,990 \\
\hline Profuturo & $3,917,169$ & $4,218,894$ & 301,725 \\
\hline SURA & $7,268,344$ & $7,682,148$ & 413,804 \\
\hline XXI Banorte & $18,186,376$ & $8,702,535$ & $-9,483,841$ \\
\hline TOTALES & $60,085,092$ & $62,374,662$ & $2,289,570$ \\
\hline
\end{tabular}

Fuente: Alto Nivel, con cifras de Consar

Imagen 5

Cuentas de AFORES

Fuente

https://www.altonivel.com.mx/finanzas/estas-son-

las-afores-ganadoras-por-numero-de-afiliados/

Por todo lo anterior, se observan caídas en el ahorro destinado a pensiones de los trabajadores de México, es entonces que se impone la pregunta: Al perderse el dinero que se ha plasmado, ¿existe vulneración de derechos humanos?, la respuesta es en sentido afirmativo, pues, el simple hecho de perder parte del ahorro de los trabajadores destinado a la pensión transgrede de manera directa el derecho a una pensión. Otra pregunta que se puede generar es al tener el Estado sólo un sistema de pensiones privado, ¿qué pasaría si las AFORES decidieran emigrar de México?, recordemos que el dinero está invertido, manejado y administrado por AFORES que son extranjeras; yla LSAR y la Comisión
Nacional del Sistema de Ahorro para el Retiro (en adelante, CONSAR) carecen de regulación para garantizar la pensión del trabajador de México.

Además, otra situación alarmante en cuanto a la vulneración de los derechos humanos en cuanto a la pensión, es que una persona que en la actualidad tiene un sueldo aproximado de $\$ 12,000.00$ (doce mil pesos) tendrá una pensión aproximada entre $\$ 2,000$ y 3,000 pesos (dos mil y tres mil pesos) (Villegas, 2015). Pues, México ha sido considerado con las aportaciones más bajas del mundo. A continuación, una estadística:

\begin{tabular}{|r|r|r|r|r|}
\hline $\begin{array}{l}\text { Salario } \\
\text { actualy }\end{array}$ & $\begin{array}{l}\text { Con } \\
\text { aportación } \\
\text { de } 6.5 \%\end{array}$ & \multicolumn{1}{l}{$\begin{array}{l}\text { Con } \\
\text { aportación } \\
\text { de } 11 \%\end{array}$} & \multicolumn{1}{l}{$\begin{array}{l}\text { Con } \\
\text { aportación } \\
\text { de } 25 \%\end{array}$} & \multicolumn{1}{l}{$\begin{array}{l}\text { Con } \\
\text { aportación } \\
\text { de } 36 \%\end{array}$} \\
\hline 10,000 & 2,600 & 3,000 & 7,000 & 10,000 \\
\hline 12,000 & 3,120 & 3,600 & 8,400 & 12,000 \\
\hline 15,000 & 3,900 & 4,500 & 10,500 & 15,000 \\
\hline 18,000 & 4,680 & 5,400 & 12,600 & 18,000 \\
\hline 20,000 & 5,200 & 6,000 & 14,000 & 20,000 \\
\hline 25,000 & 6,500 & 7,500 & 17,500 & 25,000 \\
\hline 30,000 & 7,800 & 9,000 & 21,000 & 30,000 \\
\hline
\end{tabular}

Imagen 6

Estadística aportaciones AFORE

Fuente:

https://www.elfinanciero.com.mx/economia/dimecuanto-ahorras-y-te-dire-con-cuanto-te-pensionaras

Con la estadística anterior, una pensión de 2,700 pesos en México es incapaz de mantener a una persona cuando menos con comida y servicios de su hogar, ahora que si tiene familia se vuelve imposible. 
También un hecho que cobra relevancia y que debe mencionarse es en qué invierten las AFORES a través de las SIEFORES, la Comisión Nacional del Sistema de Ahorro para el Retiro, muestra una estadística al cierre de noviembre de 2019 respecto a las inversiones, la que se plasma de la siguiente manera:

\begin{tabular}{|c|c|c|c|c|c|c|c|c|}
\hline \multicolumn{9}{|c|}{$\begin{array}{l}\text { COMPOSICION DE LAS INVERSIONES } \\
\text { Citras occentuales a l cerre de noviembre de 2019) }\end{array}$} \\
\hline \multicolumn{2}{|c|}{ Tpo Instrumento } & $\begin{array}{l}\text { Sietore } \\
\text { Bisical }\end{array}$ & $\begin{array}{l}\text { Siltore } \\
\text { Básica1 }\end{array}$ & $\begin{array}{l}\text { Sietore } \\
\text { Bisican2 }\end{array}$ & $\begin{array}{l}\text { Siltore } \\
\text { Bisisen } 3\end{array}$ & $\begin{array}{l}\text { Sietore } \\
\text { Básica 4 }\end{array}$ & $\begin{array}{l}\text { Sildore } \\
\text { Adicional }\end{array}$ & Total \\
\hline $\begin{array}{l}\text { Renta Variable } \\
\text { Nacional }\end{array}$ & $\begin{array}{l}\text { Renta Variable } \\
\text { Nacional }\end{array}$ & 0.0 & 2.1 & 5.0 & 5.7 & 7.9 & 4.5 & 5.8 \\
\hline $\begin{array}{l}\text { Renta Variable } \\
\text { Intemacional }\end{array}$ & $\begin{array}{l}\text { Renta Variable } \\
\text { Intermacional }\end{array}$ & 0.0 & 4.1 & 11.6 & 13.4 & 16.2 & 14.3 & 12.9 \\
\hline Mercancias & Mercancias & 0.0 & 0.0 & 0.2 & 0.3 & 0.3 & 0.4 & 0.2 \\
\hline \multirow{24}{*}{$\begin{array}{l}\text { Deuda Pivivada } \\
\text { Nacional }\end{array}$} & Aerolineas & 0.0 & 0.1 & 0.0 & 0.0 & 0.1 & 0.0 & 0.0 \\
\hline & Almentos & 0.0 & 0.6 & 0.9 & 0.9 & 0.8 & 0.0 & 0.8 \\
\hline & Automotiz & 0.0 & 0.1 & 0.2 & 0.1 & 0.3 & 0.1 & 0.2 \\
\hline & Banca de Desarrolo & 0.0 & 1.9 & 1.3 & 1.1 & 0.8 & 0.8 & 1.1 \\
\hline & Bancario & 0.0 & 2.1 & 1.5 & 1.3 & 1.2 & 0.9 & 1.3 \\
\hline & Bebidas & 0.0 & 0.8 & 0.6 & 0.5 & 0.5 & 0.2 & 0.5 \\
\hline & Cemento & 0.0 & 0.0 & 0.0 & 0.0 & 0.0 & 0.0 & 0.0 \\
\hline & Centros Comerciales & 0.0 & 0.1 & 0.1 & 0.1 & 0.1 & 0.0 & 0.1 \\
\hline & Consumo & 0.0 & 0.4 & 0.4 & 0.4 & 0.5 & 0.5 & 0.4 \\
\hline & Deuda CP & 0.0 & 0.0 & 0.0 & 0.0 & 0.0 & 0.0 & 0.0 \\
\hline & $\begin{array}{l}\text { Empresass productivas } \\
\text { del Estado }\end{array}$ & 0.0 & 3.6 & 2.5 & 22 & 20 & 1.7 & 2.2 \\
\hline & Estados & 0.0 & 0.8 & 0.4 & 0.3 & 0.2 & 0.4 & 0.3 \\
\hline & Europesos & 0.0 & 28 & 2.6 & 2.5 & 2.2 & 0.7 & 24 \\
\hline & Grupos Industriales & 0.0 & 0.4 & 0.3 & 0.3 & 0.2 & 0.0 & 0.3 \\
\hline & Hoteles & 0 & 0 & 0 & 0 & 0 & 0 & 0 \\
\hline & Infreestructura & 0.0 & 5.0 & 4.1 & 3.8 & 4.5 & 1.1 & 4.0 \\
\hline & Inmobialanio & 0.0 & 0.0 & 0.1 & 0.1 & 0.1 & 0.0 & 0.1 \\
\hline & OTROS & 0.0 & 0.4 & 0.5 & 0.5 & 0.6 & 0.3 & 0.5 \\
\hline & Papel & 0.0 & 0.1 & 0.1 & 0.1 & 0.0 & 0.0 & 0.1 \\
\hline & Sen. Financieros & 0.0 & 0.4 & 0.4 & 0.4 & 0.4 & 0.0 & 0.4 \\
\hline & Siderugica & 0.0 & 0.0 & 0.0 & 0.0 & 0.0 & 0.0 & 0.0 \\
\hline & Telecom & 0.0 & 1.2 & 1.2 & 1.0 & 0.9 & 0.2 & 1.0 \\
\hline & Transpotte & 0.0 & 0.5 & 0.6 & 0.6 & 0.7 & 0.0 & 0.6 \\
\hline & Nivienda & 0.0 & 2.1 & 1.3 & 1.2 & 1.1 & 0.8 & 1.2 \\
\hline Estucturado & Estocturados & 0.0 & 0.9 & 6.1 & 7.3 & 6.4 & 0.1 & 6.1 \\
\hline FIORAS & FIBRAS & 0.0 & 2.0 & 27 & 2.7 & 27 & 1.9 & 2.6 \\
\hline euda Internaciona: & Deuda intemacional & 0.0 & 2.0 & 1.4 & 1.1 & 1.4 & 0.0 & 1.3 \\
\hline \multirow{13}{*}{$\begin{array}{c}\text { Deuda } \\
\text { Gubemamental }\end{array}$} & BOND182 & 0.0 & 0.0 & 0.0 & 0.0 & 0.0 & 0.0 & 0.0 \\
\hline & BONDESO & 19.4 & 0.2 & 28 & 2.4 & 1.8 & 8.4 & 29 \\
\hline & BONOS & 1.5 & 7.8 & 11.2 & 11.1 & 126 & 16.8 & 11.4 \\
\hline & BPA182 & 6.1 & 23 & 0.8 & 0.7 & 0.6 & 0.0 & 0.8 \\
\hline & BPAS & 3.6 & 0.0 & 0.0 & 0.0 & 0.0 & 0.0 & 0.1 \\
\hline & BPAT & 0.0 & 0.0 & 0.0 & 0.0 & 0.0 & 0.0 & 0.0 \\
\hline & CBIC & 0.0 & 5.4 & 3.5 & 3.0 & 24 & 0.1 & 30 \\
\hline & CETES & 024 & 1.5 & 5.5 & 4.5 & 3.9 & 13.5 & 6.1 \\
\hline & DEPBUX & 0.0 & 0.0 & 0.0 & 0.0 & 0.0 & 0.0 & 0.0 \\
\hline & UDDBONO & 0.0 & 46.2 & 27.8 & 27.8 & 23.6 & 30.7 & 26.7 \\
\hline & UNS & 0.0 & 0.7 & 0.6 & 1.0 & 0.9 & 0.0 & 0.8 \\
\hline & REPORTOS & 6.9 & 13 & 1.7 & 1.7 & 2.1 & 1.7 & 1.9 \\
\hline & Total & 100.0 & 100.0 & 100.0 & 100.0 & 100.0 & 100.0 & 100.0 \\
\hline
\end{tabular}

Imagen 7

Instrumentos de Inversión SIEFORES

Fuente:

https://www.consar.gob.mx/gobmx/aplicativo/siset/ CuadroInicial.aspx $? \mathrm{md}=21$

Por ende, resulta importante retomar el concepto de garantías como punto principal del Derecho, pues hasta 2010 la Constitución Política de los Estados Unidos
Mexicanos, en su artículo $1^{\circ}$, habló de garantías:

“Artículo $1^{\circ}$. En los Estados Unidos Mexicanos todo individuo gozará de las garantías que otorga esta Constitución, las cuales no podrán restringirse ni suspenderse, sino en los casos y con las condiciones que ella misma establece..." (Congreso Federal, 2010).

A partir del año 2011 la Carta Magna reformó el artículo primero e incorporó a los derechos humanos:

Artículo $1^{\circ}$. En los Estados Unidos Mexicanos todas las personas gozarán de los derechos humanos reconocidos en esta Constitución y en los tratados internacionales de los que el Estado Mexicano sea parte, así como de las garantías para su protección, cuyo ejercicio no podrá restringirse ni suspenderse, salvo en los casos y bajo las condiciones que esta Constitución establece. Párrafo reformado DOF 10-06-2011 (Congreso Federal, 2019)

Por consiguiente, al constitucionalizarse los derechos humanos a partir de 2011 la Constitución Política de los Estados Unidos Mexicanos, adquirió un compromiso internacional, por lo cual el artículo 133 se relaciona con la reforma al artículo 1 , el cual reza:

Artículo 133. Esta Constitución, las leyes del Congreso de la Unión que emanen de ella y todos los tratados que estén de acuerdo con la misma, celebrados y que se celebren por el Presidente de la República, con aprobación del Senado, serán la Ley Suprema de toda la Unión. Los jueces de cada entidad federativa se arreglarán a dicha Constitución, leyes y tratados, a pesar de las disposiciones en contrario que pueda haber en las Constituciones o 
Privatization of social security in Mexico: A study of human rights' infringement

leyes de las entidades federativa. (Congreso Federal, 2019)

En consecuencia, al establecer los tratados internacionales celebrados y que se celebren por el Presidente de la República como Ley Suprema, siempre que estén acorde a la Constitución y que el Senado los apruebe; se reconoce la fuerza legal de los tratados, pero existió un problema, porque pueden existir un millón de derechos humanos (DDHH), pero sin una garantía o mecanismo que los proteja, carecen de valor jurídico.

Un ejemplo de lo anteriormente señalado es, la Convención Americana sobre Derechos Humanos suscrita en la Conferencia Especializada Interamericana sobre Derechos Humanos (B-32, San José, Costa Rica, realizada del 7 al 22 de noviembre de 1969, que en su numeral 26 señala el concepto de desarrollo progresivo, y que a la letra dice:

\section{"CONVENCION AMERICANA SOBRE DERECHOS HUMANOS (Pacto de San José) CAPITULO III DERECHOS ECONOMICOS, SOCIALES Y CULTURALES}

\begin{abstract}
Artículo 26. Desarrollo Progresivo Los Estados Partes se comprometen a adoptar providencias, tanto a nivel interno como mediante la cooperación internacional, especialmente económica y técnica, para lograr progresivamente la plena efectividad de los derechos que se derivan de las normas económicas, sociales y sobre educación, ciencia y cultura, contenidas en la Carta de la Organización de los Estados Americanos, reformada por el Protocolo de Buenos Aires, en la medida de los recursos disponibles, por vía legislativa $\mathrm{u}$ otros medios
\end{abstract}

apropiados. (Organización de Estados Americanos, 1969)

En efecto, al establecer en el Pacto de San José la efectividad en las normas sociales, se puede deducir que la realidad en nuestro país es que se carece de seguridad en cuestiones sociales, pues aunque en un contexto romántico se aprecie que el Estado buscará que un trabajador con el cambio de sistema a capitalización individual, logrará una pensión al final de sus días laborables, dicho ahorro puede ser que nunca llegue por las pérdidas que genera el sistema y que han sido evidenciadas en el presente artículo. Por tanto, si México quiere garantizar una pensión al trabajador, debe adoptar un sistema público para garantizar una pensión, pues dejar todo el recurso de los trabajadores al sector privado es un riesgo debido a que existe un lema en el argot popular, la empresa nunca pierde y es el caso en las AFORES, el que pierde es el trabajador. "Se ha dicho que una economía de libre empresa privada es inestable por esencia, que si la abandonamos a sí misma producirá ciclos recurrentes de auge y depresión”. (Friedman, 2012, pág. 49).

Entonces, la crisis financiera de 2008 produjo consecuencias graves en el Sistema de Ahorro para el Retiro así como: "La realidad es que la gran depresión, como muchos otros períodos, con un paro grave, se produjo por los errores del gobierno". (Friedman, 2012, pág. 49). Por ende, como se estableció antes, las crisis se generan por errores, por lo cual, dejar que todo el recurso del Sistema de Ahorro para el Retiro esté en manos de la iniciativa privada es un riesgo y nunca se garantizará la pensión, sólo estará en papel.

Para estar en aptitud de garantizar la pensión, debemos regresar a los juristas que hablaron de garantías individuales, tal es el caso del Maestro Ignacio Burgoa Orihuela, quien en su libro plasmó: "parece ser que la palabra "garantía" proviene del término 
anglosajón "warranty", que significa la acción de asegurar, proteger, defender o salvaguardar" (Burgoa Orihuela, 2018, pág. 161).

Entonces, al tener el derecho humano a la pensión, para lograrlo el Estado debe garantizarlo, pues en caso contrario se vulnera el derecho humano para lograr una pensión. Es importante mencionar que: "los derechos humanos son inalienables e intransferibles". (Galvis Ortiz, 2005, pág. 63), si bien es cierto que el simple hecho de pronunciar un derecho crea su existencia: "algo existe como derecho a partir del momento en que éste es pronunciado. Por ello, no es extraño que ius signifique, en ocasiones, 'actos o formalidades procesales" (Tamayo y Salmorán, 2008, pág. 12).

Sin analizar la pensión del Instituto de Seguridad Social del Estado Mexicano, que brinda dicha prestación a los trabajadores del gobierno, conocida por sus siglas como ISSSTE, la cual es materia de otra investigación, las demás AFORES que existen en México son privadas, es decir, el dinero de los trabajadores en México está en manos de la iniciativa privada, la cual genera que el Estado Mexicano omita garantizar la pensión de todos los trabajadores, pues, basta una nueva crisis financiera mundial para poner en riesgo los recursos de los trabajadores.

Además, otra situación alarmante o que evidencia que el ahorro de los trabajadores destinado a la pensión está en juego, es:

...ocho de cada 10 pesos de los recursos captados este viernes en el mercado bursátil mexicano para financiar la construcción del Nuevo Aeropuerto de la Ciudad de México (NAICM) fueron contratados por las empresas que gestionan los fondos de pensión de los trabajadores, dijo a La Jornada Ricardo Rangel Fernández Mc
Gregor, de CI Banco, la institución financiera... (La Jornada, 2018, párrafo primero)

Por tanto, es menester señalar que actualmente está detenida la construcción de dicho proyecto desde la toma de protesta del nuevo Presidente de la República en México, pero se presentó otro proyecto de obra pública que es un tren turístico en la zona Maya y también se puede financiar con el dinero del Sistema de Ahorro para el Retiro.

Es decir, el recurso destinado a pensiones está invertido y es un riego que el fondo destinado a pensiones esté invertido, en otro supuesto, qué pasaría si alguna de las AFORES emigrara de México, basta visualizar para entender que la privatización de pensiones vulnera derechos humanos.

\section{CONCLUSIÓN}

Después del análisis a partir de la crisis financiera de 2008, se evidenció en México que las AFORES carecen de una garantía para resguardar el capital destinado a la pensión de los trabajadores, por tanto, hay vulneración al derecho humano del trabajador con su cuenta de retiro.

Por consiguiente, el recurso destinado a pensiones está en riesgo, depende de entes financieros privados y de capital extranjero. Como se citó en el documento, dejar todo en manos de la iniciativa privada es un riesgo para los gobernados, pues se carece de garantías para el cumplimiento de generar una pensión, basta que los bancos emigren de México con el dinero de los trabajadores o bien se pierda el recurso en las inversiones, es decir, la AFORE administra y la SIEFORE invierte el dinero de los trabajadores, nunca estos entes financieros arriesgan su capital propio, pues sólo está en juego el dinero de sus derechohabientes, ya en el estudio se dio la 
Privatization of social security in Mexico: A study of human rights' infringement

cifra de los derechohabientes que existen en México.

Por último, quedó demostrado que a partir de 2008, la crisis generó grandes pérdidas, las cuales son solo para los trabajadores, quienes todos los días invierten derivado de su trabajo a su cuenta de ahorro para el retiro y los entes financieros invierten y prestan los recursos como si fueran propios, pues la nula regulación hacia estos entes les permite jugar y especular con el recurso.

\section{PROPUESTA}

México debe seguir el modelo de Perú al considerar un sistema público de pensiones, que permita al trabajador optar por el sector privado o público para administrar su ahorro para el retiro.

Por consiguiente, aunque en apariencia el sector público tendrá un rendimiento menor al privado, los riesgos son mínimos, en cambio si todo está en manos del sector privado, con las inversiones y las pérdidas se vulnera el derecho humano de los trabajadores para lograr su pensión, pues puede darse el caso en que al momento de pensionarse un trabajador, el Sistema de Ahorro para el Retiro carezca de fondos para pagar, además, de que la legislación en México es débil para sancionar estos hechos.

Lo anterior se ha visto en nuestro país, pues se ha subido la edad para pensionarse y la realidad es que se carece de recursos para cubrir la pensión de todos los trabajadores de México, entonces aumentar la edad de pensión se vuelve altamente riesgoso.

En México con la adecuación de un sistema de pensión pública, se garantizará el acceso a ella, pues el trabajador, tendrá la opción de elegir entre la iniciativa privada o la pública, ahí se dejaría de vulnerar el derecho humano de acceso a la pensión.

\section{FUENTES DE INFORMACIÓN}

\section{Fuentes electrónicas}

La Jornada. (24 de Marzo de 2018). jornada.com, On Line. Recuperado de https://www.jornada.com.mx/ultimas/2018 /03/24/financian-nuevo-aeropuerto-condinero-de-afores-9601.html Consultada 26/10/2018.

Congreso Federal. (1917). Cámara de Diputados. Recuperado de http://www.diputados.gob.mx/LeyesBiblio/ ref/dof/CPEUM_orig_05feb1917_ima.pdf

Congreso Federal. (1929). Cámara de Diputados. Recuperado el 03 de Diciembre de 2019, Recuperado de http://www.diputados.gob.mx/LeyesBiblio/ ref/dof/CPEUM_ref_008_06sep29_ima.pd $\mathrm{f}$

Congreso Federal. (1995). Diario Oficial de la Federación, Tomo DVII $N^{\circ} 16$. Recuperado de http://www.ordenjuridico.gob.mx/Docume ntos/Federal/pdf/wo9056.pdf

Congreso Federal. (2010). Secretaria de Trabajo y Previsión Social. Recuperado de http://www.stps.gob.mx/bp/secciones/dgsst /normatividad/1.pdf

Congreso Federal. (2019). Camara de Diputados. Recuperado de http://www.diputados.gob.mx/LeyesBiblio/ pdf/1_201219.pdf

Albarrán, E. (2018). Cuatro Afores invierten en el nuevo aeropuerto. El Economista, págs. https://www.eleconomista.com.mx/mercad os/cuatro-afores-invierten-en-el-nuevoaeropuerto-20180327-0093.html.

BBC Mundo. (27 de Enero de 2011). BBC. Recuperado de https://www.bbc.com/mundo/noticias/2011 /01/110127_economia_eeuu_crisis_evitabl e jrg 
Burgoa Orihuela, I. (2018). Las garantías individuales (41 ed.). Mexico, México: Porrúa. Recuperado el 26 de Octubre de 2019

Carrillo, R. d. (07 de Noviembre de 2018). El Heraldo. Recuperado de http://www.heraldo.mx/sufren-aforespeores-minusvalias-del-sexenio/

Friedman, M. (2012). Capitalismo y Libertad. Madrid, España: Sintesis. Recuperado el 26 de Octubre de 2019

Galvis Ortiz, L. (2005). Compresión de los Derechos Humanos (Tercera, actualizada ed.). Bogota, Colombia: Ediciones Aurora. Recuperado el 04 de Enero de 2020

Garduño, R. M. (26 de Marzo de 2009). Pérdidas en los ahorros de trabajadores por 147,850 millones de pesos en 23 meses. La Jornada-Economía. Recuperado el 02 de Febrero de 2020, de https://www.jornada.com.mx/2009/03/26/e conomia/027n1eco

Gutierrez Ayala, M. (2017). El Derecho Laboral el poder económico y la apariencia de un buen derecho. En Aspectos Laborales en e siglo XXI (pág. 89). Puebla, México: Dirección de Fomento Editorial.

Kurczyn Bañuelos, S. (Septiembre de 1996). Reforma del sistema de pensiones mexicano: principales aspectos macroeconómicos. (Bancomext, Ed.) Revista Bancomext, 741. Recuperado de http://revistas.bancomext.gob.mx/rce/maga zines/324/10/RCE10.pdf

Martinez Gonzalez, M. d. (17 de Mayo de 2015). Frecuencia Laboral, Año 9. (Portal Semanario). Recuperado de http://www.frecuencialaboral.com/aforespe rdidasmillonarias2015.html
Meléndez George, L. M. (2011). Los retos del sindicalismo mexicano. (B. U. Puebla, Ed.) Puebla, México: Dirección de Fomeno Editorial.

NOTIMEX. (20 de Mayo de 2019). El Economista. Recuperado el 02 de Febrero de 2020, de https://www.eleconomista.com.mx/sectorfi nanciero/Las-afores-con-mayoresperdidas-de-cuentas-de-ahorro-al-cierrede-abril-segun-la-Consar-201905200043.html

Organización de Estados Americanos. (7-22 de Noviembre de 1969). Organización de Estados Americanos. Recuperado de https://www.oas.org/dil/esp/tratados_b32_convencion_americana_sobre_derecho s_humanos.htm

Sandoval, A. (22 de Noviembre de 2018). Alto Nivel. Recuperado de https://www.altonivel.com.mx/finanzas/est as-son-las-afores-ganadoras-por-numerode-afiliados/

Tamayo y Salmorán, R. (2008). Introducción Analítica al Estudio del Derecho. México, México: Themis. Recuperado el 26 de Octubre de 2019

Torres, Y. (07 de Julio de 2013). Afores, con las minusvalías más grandes de la historia. El economista. Recuperado de https://www.eleconomista.com.mx/sectorfi nanciero/Afores-con-las-minusvalias-masgrandes-de-la-historia-201307070064.html

Torres, Y. (07 de Julio de 2013). El economista. Recuperado el Marzo de 2020, de El economista: https://www.eleconomista.com.mx/sectorfi nanciero/Afores-con-las-minusvalias-masgrandes-de-la-historia-201307070064.html 
Privatización del sistema de seguridad social en México: Un estudio de la vulneración a los derechos humanos

Privatization of social security in Mexico: A study of human rights' infringement

Villegas, D. (2016 de Octubre de 2015).

Recuperado de

https://www.elfinanciero.com.mx/economi

a/dime-cuanto-ahorras-y-te-dire-con-

cuanto-te-pensionaras 